\title{
Aerodynamic Noise Characteristics of Horizontal Axis Wind Turbine Blade Using CAA
}

\author{
Kyoungsoo Lee, Ziaul Huque, Raghava Kommalapati, Shrabanti Roy, Chao Sui, and Nazia Munir
}

\begin{abstract}
This paper presents the aero-acoustic characteristics of horizontal axis wind turbine blade using computational aero-acoustic. The National Renewable Energy Laboratory (NREL) Phase VI wind turbine blade configuration was considered for the studies. This paper aims at predicting the sound noise generated by rotating wind turbine using unstructured mesh. The LES simulation was used for unsteady transient simulations with hybrid FW-F technique for mid to far-field aero-acoustic sound noise simulation. The volumetric quadrupole noise source was considered to estimate the rotating effect of rotor blade even though the simulation is low Mach number. To perform the numerical simulation for noise characteristics of rotating wind blade, rigid body motion was imposed to the blade surface. The surface total noise term, which includes the thickness and pressure loading terms, and volume quadratic term are obtained efficiently for reference and rotating frame motions respectively. However significant discrepancy of volume and total sound noise level was obtained between reference and rotating motion cases. The unsteady rotating effects of wind blade on the sound noise emission could be simulated well using the process of this study.
\end{abstract}

Index Terms-NREL phase VI, wind blade, aero-acoustic, aerodynamic, CAA.

\section{INTRODUCTION}

Wind energy is a popular form of renewable energy due to its low air pollution compared to other conventional thermal power plants. It reduces the use of conventional fossil fuel and the emission of the gases responsible for the green house effect. But there are still several issues which need to be resolved in order to increase its efficiency and mitigate the effects of some of the disadvantages like noise radiated from the rotating Horizontal-Axis Wind Turbine (HAWT) [1]-[4], especially the aerodynamic noise emitted from the wind turbine blades. The power generating efficiency of wind blade is highly sensitive to the blade shape parameters (such as the airfoil shape, twist angle, pitch angle, tip shape etc.) and free stream wind speed. It was recognized as one of main design objectives for recent commercial wind turbine blades. Besides, the noise controlled wind blades are challenging and ongoing for past the three decades [5]-[11].

Manuscript received February 20, 2015; revised August 6, 2015. This research was supported by the National Science Foundation (NSF) through the Center for Energy and Environmental Sustainability (CEES), a CREST Center (Award No. 1036593).

Kyoungsoo Lee is with Center for Energy and Environmental Sustainability (CEES), Prairiew View A\&M University, Prairie View, TX, 77446, USA (e-mail: kylee@pvamu.edu).

Ziaul Huque, Raghava Kommalapati, Shrabanti Roy, Chao Sui, and Nazia Munir are with the Mechanical Engineering Department, CEES, Prairie View A\&M University, Prairie View, TX, 77446, USA (e-mail: \{zihuque, rrkommalapati, rshrabanti, csui, nmunir\}@pvamu.edu).

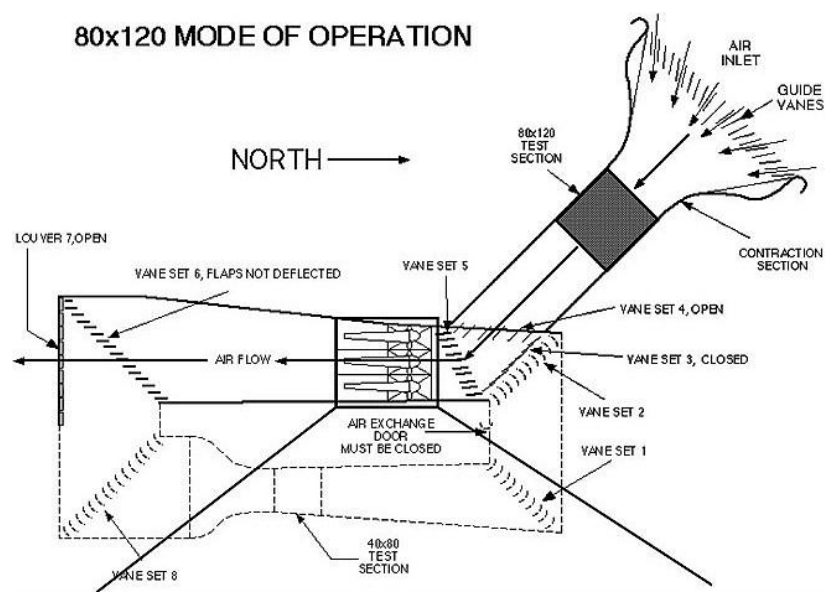

(a). NASA Ames research centre full scale aerodynamic complex.

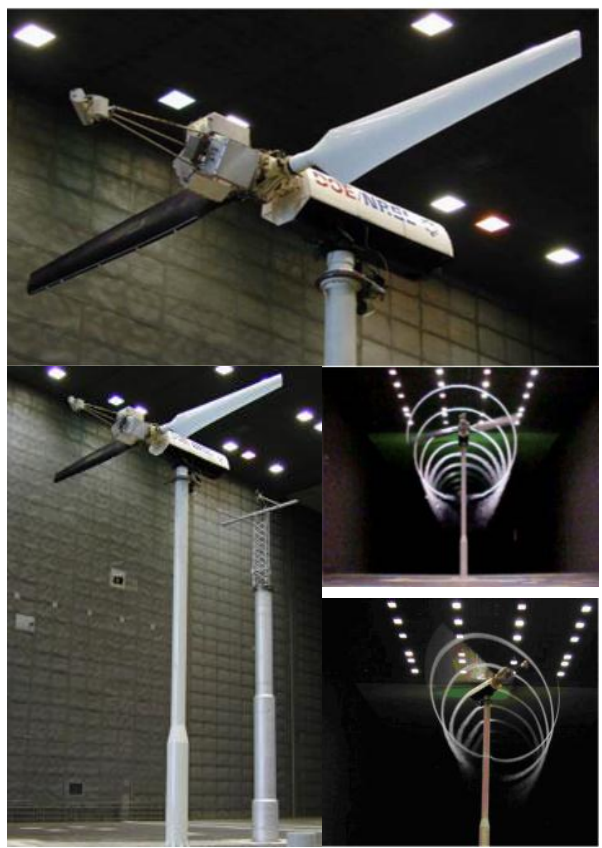

(b). Full scale NREL phase VI wind turbine blade.

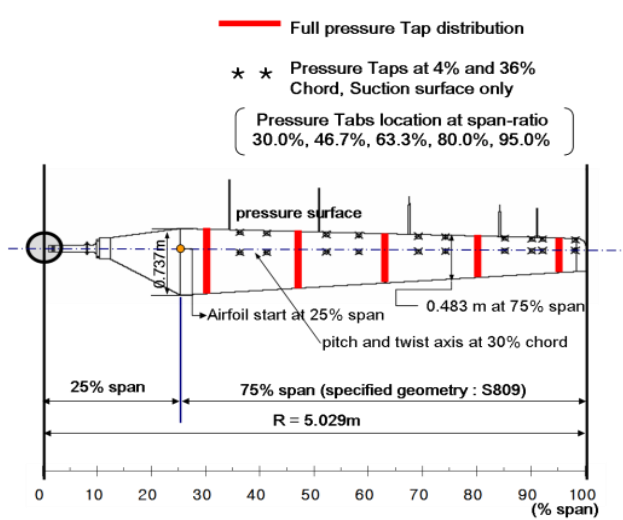

(c). Pressure taps distribution along the span.

Fig. 1. NASA Ames research center full scale aerodynamic complex: NREL phase VI wind turbine blade [3], [4]. 
This paper aims at predicting the noise characteristics generated by flows interacting with wind turbine blade using Computational Aero-Acoustics (CAA) [12]-[15]. To this end, the unstructured Large-Eddy simulation (LES) coupled with a hybrid integral Ffowcs Williams-Howkings (FW-F) technique was used for far-field noise prediction using commercial CFD software STAR-CCM+ [16]. It can resolve the tonal and the broadband components of the acoustic pressure spectrum, while the FW-H analogy enables the efficient propagation of the pressure in the acoustic far-field without the numerical dispersion of a numerical scheme. The surface and volumetric noise source terms are considered from the pressure recorded result, and the surface noise term is consisted by surface loading and thickness terms. Even though, low-Mach number nature of wind turbine blade which is governed by the aerodynamic characteristics, the quadratic volumetric noise sound level is calculated in addition to the surface sound noise.

To perform the numerical simulation of wind blade noise characteristics, the initial aerodynamic fluid field domain was determined using steady 3D Computational Fluid Dynamics (CFD) with RANS simulation for the well known National Renewable Energy Laboratory (NREL) Phase VI wind blade [1]-[4] of Fig. 1 which uses S809 airfoil profile for section. The extensible experimental [1]-[4] and numerical data were reported by many researchers [17]-[23] to demonstrate the accuracy of CFD for aerodynamic problem. After determining initial steady state, the unsteady LES simulations were performed to predict mid to fair field noise level considering rigid body motion for rotating blade. The rotational frame motions are considered to consider the effects of rigid body motion of rotating blade. The free-stream $25 \mathrm{~m} / \mathrm{s}$ wind speed and 72RPM are considered for the free-stream wind and rotating speeds.

\section{NOISE SOURCE OF WIND TURBINE BLADE}

The wind turbine produces the electricity by the help of aerodynamic force of wind blade. To increase the power generating efficiency and aerodynamic characteristics the optimized wind blade shape has to be determined. The noise of wind turbine system mainly initiated from the mechanical and aerodynamic source. Mechanical noise is caused by gears and bearings. It has been reduced considerably over the past years and is becoming less of a concern. It can be kept within limits by proper insulation around the gearbox. To further reduce wind turbine noise, focus must be placed on aerodynamic noise. Between them the aerodynamic noise can be regarded as the main focus which is considered the most significant form of noise and the most difficult to address in wind turbine.

Aerodynamic noise can be distinguished, notably low frequency (tonal) noise and high frequency broadband noise. The tonal noise is generally low frequency and due to the disturbance in the flow caused by the movement of rotating blade (thickness noise) and associated pressure field (loading noise). The broadband noise is higher frequency and due to various types of turbulent flow interaction with the blades [11]. The aerodynamic sound noise emitted by wind turbines can have a significant impact on the population residing near the wind turbines. The installation of wind turbines is becoming a serious problem in countries with high population density as a result of wind turbines producing aerodynamic noise. The noise of wind turbines is a major problem for the public acceptance of wind energy. Noise is becoming a serious concern early in the design process rather than a problem to be corrected during the production or testing stages.

The sound noise level emitted from the wind turbine blade can be classified as the aero-acoustic field. And the aero-acoustics investigates the sound noise generated from the aerodynamic characteristics of wind blade near the surface. Before the aero-acoustic science was developed, the flow-generated noise studies were focused on the relation between the frequency of the fluid fluctuations and the emitted sound. Decades ago, the aero-acoustics science was initiated to develop a theory for the estimation of the intensity of sound that radiates from a turbulent flow by Lighthill [12]. Lighthill established the theoretical background generally referred to when investigating aerodynamic noise. Lighthill first introduced the concept of aeroacoustic analogy which consists of replacing the actual flow field responsible for generating noise with an equivalent system of noise sources. The noise sources act on a uniform stagnant fluid that is governed using standard acoustic propagation equations. The aerodynamic characterization of the sources then becomes the main issue in noise prediction. The Lighthill analogy was extended to account for arbitrary surface motion by Ffowcs Williams and Hawkings [13]. This formulation was used for noise prediction in rotor blades aerodynamics, such as in the helicopter and turbine industries [14], [15].

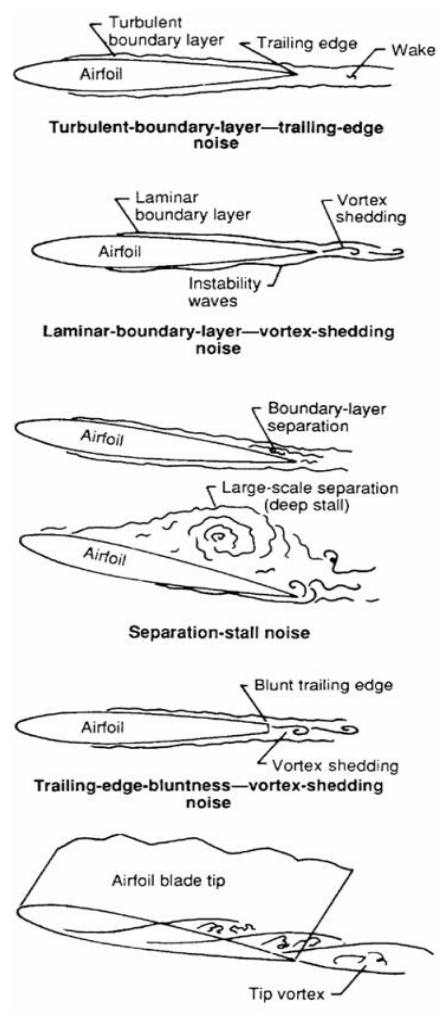

Tip vortex formation noise

Fig. 2. Airfoil self noise [10].

The main mechanisms of sound generation in the presence of solid structures of wind blade can be classified as vortex shedding noise, turbulence-structure interaction noise, trailing edge noise as shown in Fig. 2. The vortex shedding 
noise is related the vorticity that is released from a bluff body in a flow. The time varying circulation on the body due to vortex shedding induces a fluctuating force on the body itself which is transferred to the fluid and propagates as sound. Vortical structures impinging on a solid surface generate local pressure fluctuations on the body surface which feed the acoustic far field and produce the turbulence-structure interaction noise. The trailing edge noise is important for all rotating blade technologies, due to the interaction of the boundary layers instabilities with the surface edges. In Fig. 3 shows the vorticity results of this study for NREL Phase VI wind turbine blade in the $7 \mathrm{~m} / \mathrm{s}$ wind speed case. From Fig. 3, it can be understood that the wake of trailing edge and tip region are important source for sound noise.

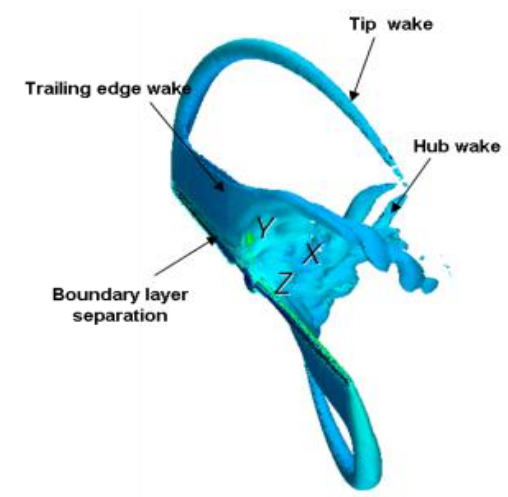

Fig. 3. Rotating NREL phase VI wind blade: $7 \mathrm{~ms} / \mathrm{s}$ wind speed case.

\section{NREL PHASE VI WIND TURBINE BLADE}

The aero-acoustic sound noise is highly dependent to the aerodynamic characteristics. So the evaluation of aerodynamic load and its verification are necessary and important. To simulate the sound noise level of rotating wind turbine, the blade profile of NREL Phase VI wind blade is adopted. There is comprehensive information about the unsteady aerodynamic experiment of it, and its primary objective was to provide information needed to quantify the full scale, 3D aerodynamic behavior of horizontal-axis wind turbines (HAWT). From the field-testing, it has shown that 3-D effects are prevalent in wind turbine field operation, the wind turbines undergo very complex aerodynamic response phenomena when operating in the field environment. To provide the needs of accurate $3 \mathrm{D}$ aerodynamic force which is considering the inflow turbulence and shear across the rotor plane, the wind tunnel test was performed by NREL using Phase VI wind blade. From the rigorous experimental wind tunnel test, accurate quantitative aerodynamic and structural measurements on a full scale wind turbine blades were acquired.

As shown in Fig. 4, the wind turbine blade of NREL Phase VI is based on the S809 airfoil (Fig. 4(a)) with $10.058 \mathrm{~m}$ diameter rotor, and is both nonlinearly twisted and linearly tapered [3]. The blade chord tapered from $0.737 \mathrm{~m}$ at $0.25 \mathrm{R}$ to $0.356 \mathrm{~m}$ at the tip. Blade section twist decreased from $20.0^{\circ}$ at $0.25 \mathrm{R}$ to $-2.0^{\circ}$ at the tip. Between $0.25 \mathrm{R}$ and the tip, blade cross-section was uniform, corresponding to the S809 airfoil. The airfoil section at $0.25 \mathrm{R}$ was joined to the pitch shaft section at $0.12 \mathrm{R}$ using linear segments to yield an uninterrupted transition between these two disparate contours
The blade pitched about an axis located 0.30-chord of the leading edge, and centered between the blade upper and lower surfaces at that chord location.

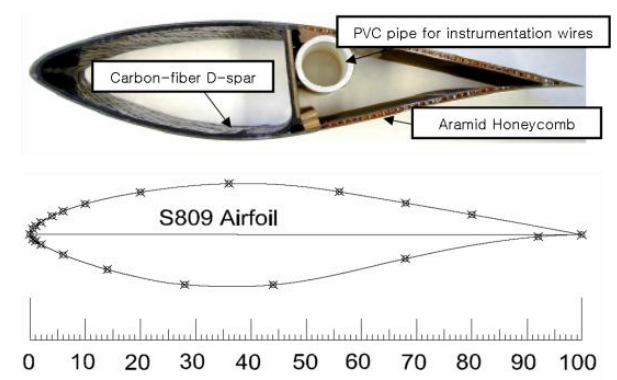

(a) Pressure tab distribution.

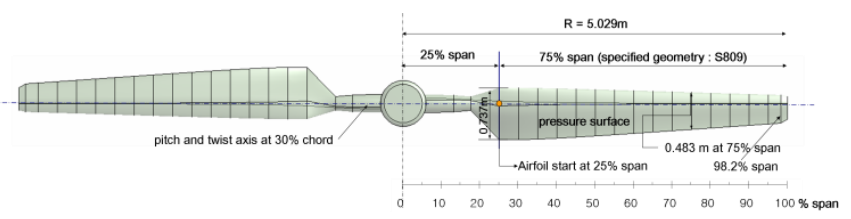

(b) NREL Phase VI wind blade.

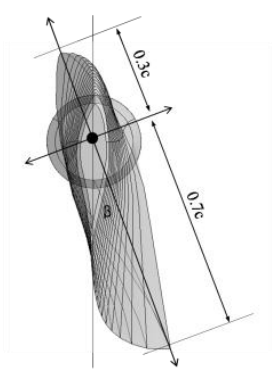

(c) Section of twist angle.

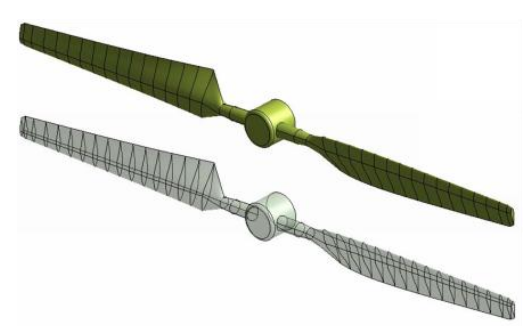

(d) Isometric view.

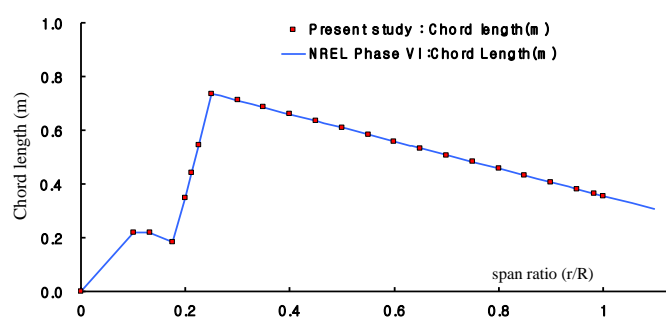

(e) Twist angle distribution along span ratio.

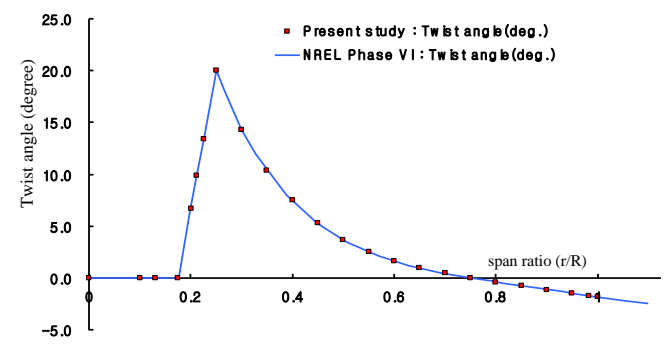

(f) Taper (chord length) distribution along span ratio. Fig. 4. NREL phase VI wind blade configuration. 


\section{AERODYNAmic Force Evaluation}

The aero-acoustic characteristics of wind turbine are highly dependent to the aerodynamic force on the blade surface. To verify the CFD analysis for the aerodynamic force of NREL Phase VI wind turbine, the aerodynamic force of it was evaluated and verified with steady state CFD analysis.

Fig. 5 and Fig. 6 show the aerodynamic characteristic of global torque (J) and thrust force $(\mathrm{N})$ and are compared with the reported results. The SST turbulent model was used for the steady state simulation with fully turbulent option. The results are good agreement with NREL's experiment. The results obtained from the other methods are also compared. In Fig. 5, a comparative view of current study with the NREL experimental values and values obtained from the other studies. From reference [17]-[23] it is observed that various approaches have been taken by the researchers for evaluating aerodynamic forces of wind turbine. In 2003 Duque et al. applied Vortex Lattice and stall delay method to determine the aerodynamic forces [17] while Tongchitpakdee et al. uses both Boldwin-Lomax turbulence model and Spalart-Allmaras model [18]. In 2012 Mo and Lee uses fluent and SST model [20] while Yelmule and Anjuri uses CFX and SST model [23]. All the results of references and NREL experimental values are presented in Fig. 5 with the current study. It is observed that the present study shows good agreement with NREL experimental values than the values of other studies.

The thrust force of Fig. 6 shows that current results are higher than NREL in lower wind speed cases $(5-10 \mathrm{~m} / \mathrm{s})$. After $13 \mathrm{~m} / \mathrm{s}$ wind speed, the thrust force is less than NREL. However the overall thrust force results are good agreement with NREL and Yelemule and Anjuri [23]. Since, the accuracy of aerodynamic force which obtained by the CFD analysis was verified.

The torque is directly related to the wind turbine power generation ratio. As shown in Fig. 5, the amount of torque result is not proportional to the wind speed. Even though the wind speed is increasing, the toque value decreases in specific region of $13-15 \mathrm{~m} / \mathrm{s}$. And it is increasing after $20 \mathrm{~m} / \mathrm{s}$ wind speed. This phenomenon is known the stall effect of wind turbine blade comes from the 2D airfoil. So, the power generation efficiency of wind turbine is highly related to the relationship of aerodynamic force and stall in airfoil and blade. The results of Blade element momentum (BEM) and Vortex lattice method (VM) shows large difference from the NREL's wind tunnel test data after $10 \mathrm{~m} / \mathrm{s}$ wind speed case. Before $10 \mathrm{~m} / \mathrm{s}$ wind speed, BEM and VM show good agreement with CFD [17]-[24] wind tunnel test [3] and this study. However the results of stall region $(13-15 \mathrm{~m} / \mathrm{s})$ and after stall region $(20-25 \mathrm{~m} / \mathrm{s})$ that over-estimate the torque in Fig. 5. The BEM and VM commonly use the aerodynamic force coefficient which obtained from 2D airfoil wind tunnel test. When BEM and VM use 2D aerodynamic force coefficient for 3D NREL Phase VI wind blade, BEM and VM can produce un-correct aerodynamic force in stall region.

Fig. 7 shows the vorticity solution according to the wind speed cases from $5 \mathrm{~m} / \mathrm{s}$ to $25 \mathrm{~m} / \mathrm{s}$. As the speed increase, the vorticity near the blade surface and downsteam region are increase. Before $10 \mathrm{~m} / \mathrm{s}$ wind speed, there are little wake and turbulence.

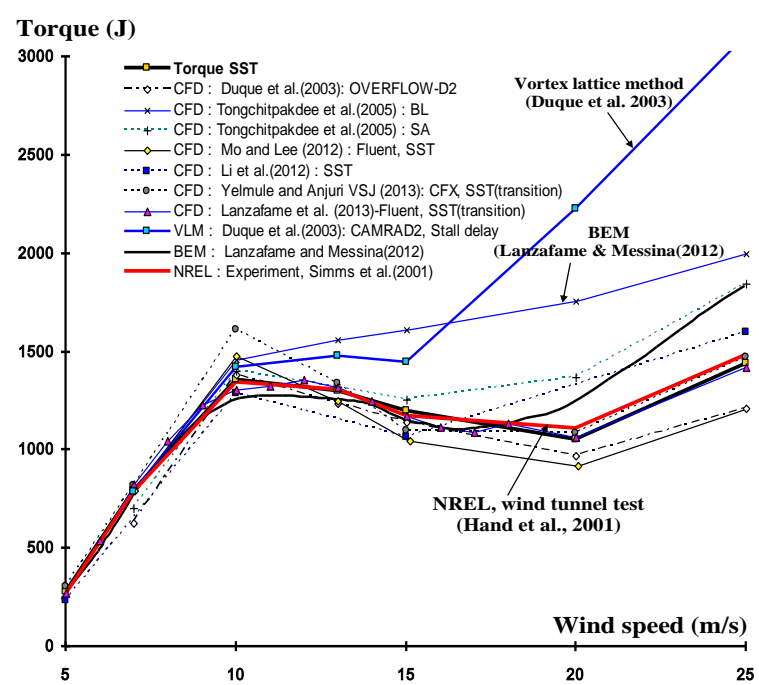

Fig. 5. Aerodynamic force: Torque (J).

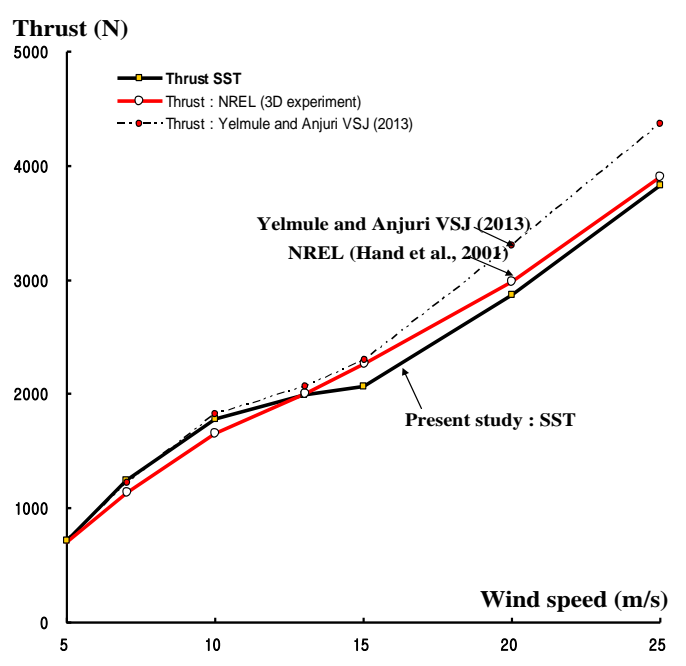

Fig. 6. Aerodynamic force: Thrust (N).
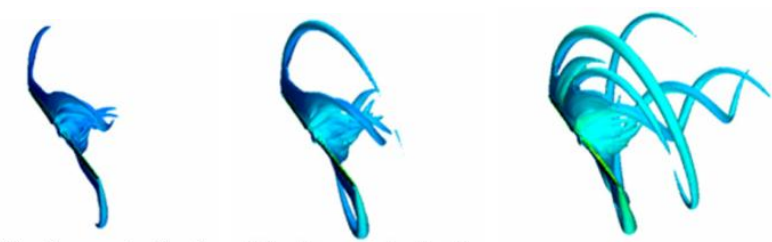

Wind speed : $5 \mathrm{~m} / \mathrm{s}$ Wind speed $: 7 \mathrm{~m} / \mathrm{s}$ Wind speed $: 10 \mathrm{~m} / \mathrm{s}$

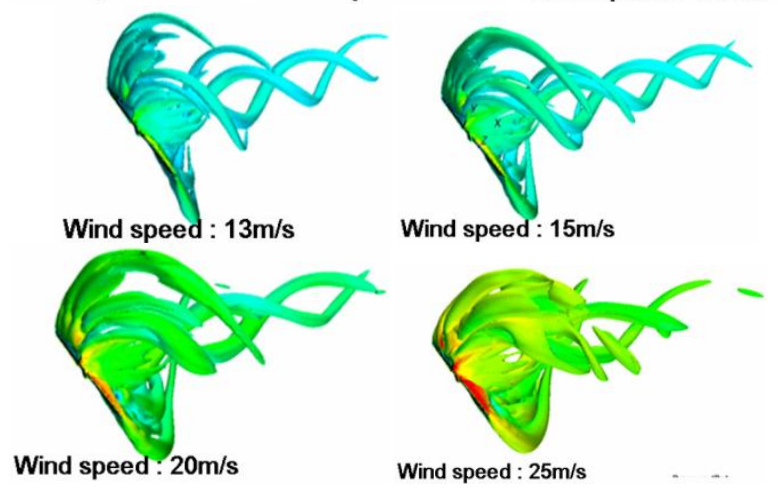

Fig. 7. Vorticity results according to the wind speed: Steady state.

\section{CAA RESULTS AND DISCUSSION}

To compute the location and strength of the main noise sources, the broadband noise source models can be used in near-field noise prediction. The noise sources consist of a volume distribution of quadrupole sources and a surface 
distribution of dipole sources. STAR-CCM+ [16] offers a group of models capable of evaluating the broadband sources of noise. In the frequency domain, a broadband noise has a continuous spectrum, where the acoustic energy is continuously distributed at all frequencies in a given range. By using the broadband models, it can compute the location and strength of the main sources of noise that are generated aerodynamically. That use flow field quantities from Reynolds-Averaged Navier-Stokes (RANS) equations.

Modern CFD adopts two strategies for prediction of mid-field to far-field acoustic prediction: one is based on the Direct Noise Simulation of sound propagation and the other is based on integral formulations. Both approaches are known Computational Aero-Acoustics (CAA) methods, and have been extensively used to study the sound generation and sound propagation in the fluid flow. Direct simulation can be too expensive to predict noise levels some distance from the source. The FW-H acoustics integral can be used with less computational cost within accuracy for mid to far-field noise predictions with sound signal radiated from near-field flow data from CFD solution. The steady and unsteady CFD solutions are possible to predict the noise source. But the steady solution can not account the time dependent terms and quadrupole noise source which is important and must be considered when the high shear layer and Mach number.

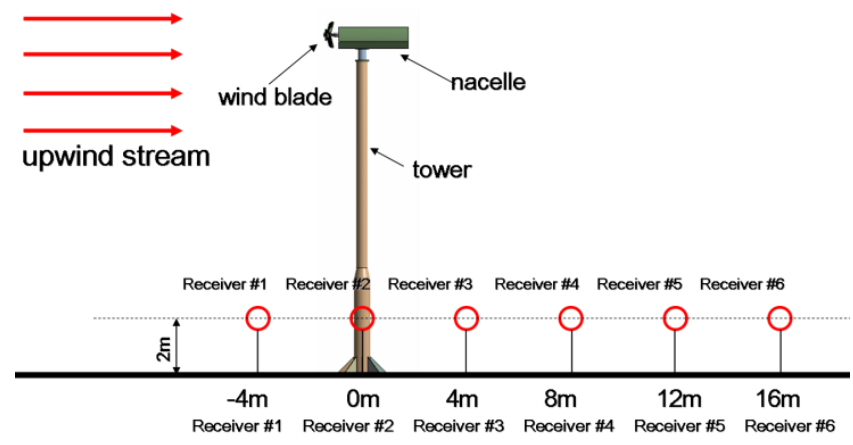

Fig. 8. FW-H receiver locations.

The FW-H integral formulation is essentially an inhomogeneous wave equation that can be derived by manipulating the continuity equation and the Navier-Stokes equations. If non-convection form of FW-H of Farassat's Formulation is adopted, the FW-H equation for pressure that is radiated into a medium at rest by a flow in a region or a set of surfaces can be written as

$$
p^{\prime}(\vec{x}, t)=p_{T}^{\prime}(\vec{x}, t)+p_{L}^{\prime}(\vec{x}, t)+p_{Q}^{\prime}(\vec{x}, t)
$$

where, $p_{T}^{\prime}(\vec{x}, t), p_{L}^{\prime}(\vec{x}, t), p_{Q}^{\prime}(\vec{x}, t)$ are terms of monopole, dipole and quadrupole respectively. When the integration surface coincides with the body, these terms are called as: "Thickness Surface Term", "Loading Surface Term" and "Volume Term" respectively. The volumetric qudrupole noise sound effect can be omitted in the case of low Mach number or little shear layer effect conditions.

The FW-H 6-pointer receivers for noise prediction are located in the center line below wind blade as shown in Fig. 8 To account for the unsteady blade motions, the rigid body rotating motion is considered to the wind blade. From the wind tunnel test condition, the rotating speed is constant 72
RPM. The time dependent LES vorticity results re shown in Fig. 9 for different wind speed cases. And Fig. 10 shows the time varying vorticity results in $25 \mathrm{~m} / \mathrm{s}$ wind speed case at different time steps.

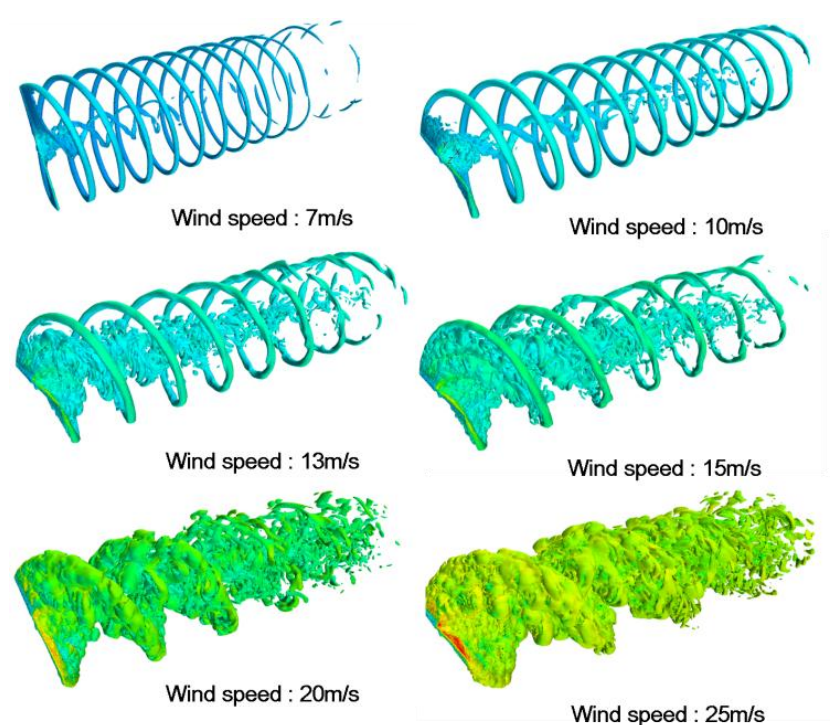

Fig. 9. Vorticity results according to the wind speed: LES.
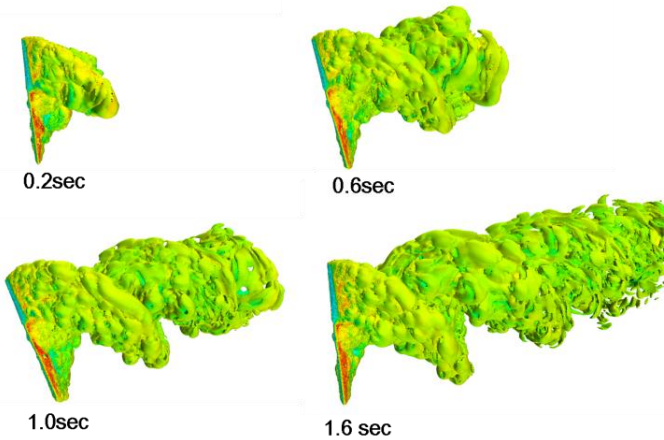

Fig. 10. 25m/s wind speed case: LES.

And the surface total sound pressure level (SPL, $\mathrm{dB}$ ) noise predictions are plotted in Fig. 11 when $25 \mathrm{~m} / \mathrm{s}$ upwind wind speed applied for surface (dipole) and volume (quadrupole) according to the frequency region. Both tonal low frequency and broadband high frequency sound noise perceived well by the $\mathrm{FW}-\mathrm{H}$ receivers.

In Fig. 11, thickness surface term (monopole), loading surface term (dipole) and volume term (quadrupole) in equation (1) were predicted independently. The thickness term is less than surface loading term, but the volumetric term is much higher than surface term. The rigid body motion wind turbine blade was considered from rotating frame motion. It looks over-estimate the sound noise level. Low frequency tonal noise of thickness and surface loading terms can be produced by the disturbance in the flow caused by the movement of rotating blade (thickness noise) and associated pressure field (loading noise). The broadband noise is higher frequency and due to various types of turbulent flow interaction with the blades. From the study of Fig. 11, the thickness surface tem is relatively low sound level in low frequency region. However the low frequency tonal sound noise is higher than high frequency broadband noise for surface loading term. The surface total term was determined by summation of thickness and load term on surface. The thickness term has little effect on the surface total sound 
noise level. In future study, the accuracy and effects of volumetric quadrupole sound noise level will be studied.
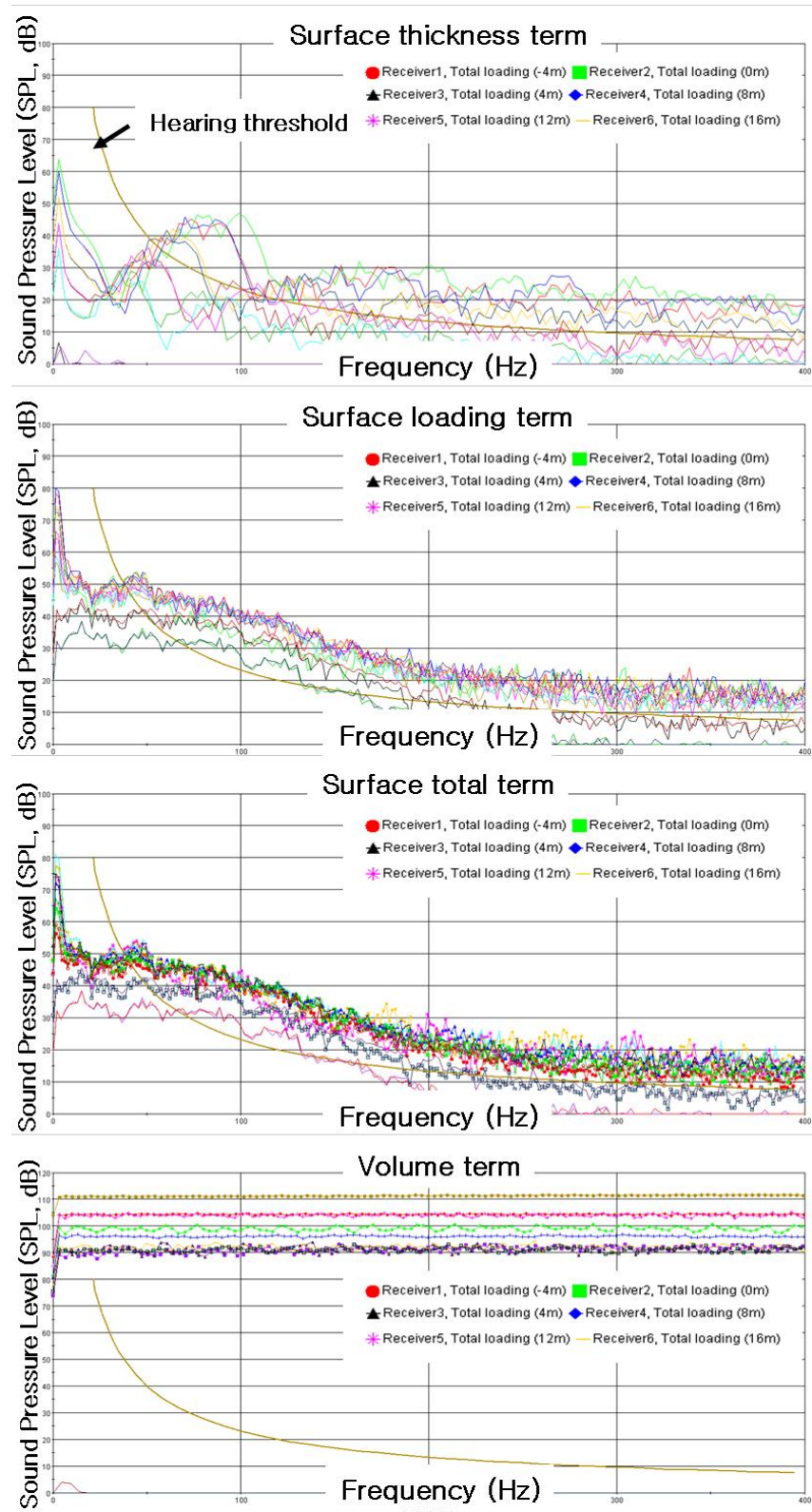

Fig. 11. Surface and volume mid to far-field sound level (dB).

\section{CONCLUSION}

The far-field noise predictions of NREL Phase VI wind blade were simulated for surface noise level using unsteady DES coupled with a hybrid integral FW-F technique. The commercial CFD software STAR-CCM+ was adopted. To account the more exact and realistic time dependent unsteady air pressure on the noise source, the rigid body motion was considered to the wind blade. The surface total noise term which including the thickness and pressure loading terms are obtained efficiently. The surface loading term which produced by the unsteady motion of the force distribution on the body surface is more significant than the thickness term which produced by displacement of fluid of rotating blade. The volumetric quadrupole noise source was also considered and shows far higher than surface loading term, even though wind turbine's low Mach number. The unsteady rotating effects of wind blade on the noise radiation can be simulated well using the process of this study. The tonal and the broadband components of the acoustic pressure spectrum were resolved successfully, while the FW-H analogy enables the efficient propagation of the pressure in the acoustic far-field without the numerical dispersion of a numerical scheme.

\section{REFERENCES}

[1] R. R. Ramsay, M. J. Hoffman, and G. M. Gregorek, "Effects of grit roughness and pitch oscillation on the S809 airfoil," NREL/TP-442-7817, National Renewable Energy Lab., 1995.

[2] D. M. Sommers, "Design and experimental results for the S809 airfoil," NREL/SR-440-6918, National Renewable Energy Lab., January 1997.

[3] M. Hand, D. Simms, L. J. Fingersch, D. Jager, S. Larwood, J. Cotrell, and S. Schreck, "Unsteady aerodynamics experiment phase VI: Wind tunnel test configurations and available data campaigns," NREL/TP-500-29955, National Renewable Energy Lab., December 2001.

[4] D. Simms, S. Schreck, M. Hand, and L. J. Fingersch, "NREL unsteady aerodynamics experiment in the NASA-Ames wind tunnel: A comparison of predictions to measurements," NREL/TP-500-29494, National Renewable Energy Lab., June 2001.

[5] A. Bruijn, W. J. Stam, and W. J. Wolf, "Determination of the acoustic source power levels of wind turbines," in Proc. the European Wind Energy Conference, Hamburg, Germany, 1984.

[6] H. H. Hubbard and K. P. Shepherd, "Aeroacoustics of large wind turbines," J. Acoust. Soc. Am., vol. 89, no. 6, pp. 2495-2508, 1991.

[7] G. Guidati, J. Ostertag, and S. Wagner, "Prediction and reduction of wind turbine noise: An overview of research activities in Europe," AIAA Paper 2000-0042, 2000.

[8] F. B. Matthew, "Survey of techniques for reduction of wind turbine blade trailing edge noise," SAND2011-5252, Sandia National Lab., August 2011.

[9] S. Wagner, R. Bareib, and R. G. Guidati, Wind Turbine Noise, 1996.

[10] T. F. Brooks, D. S. Pope, and M. Z. Marcolini, "Airfoil self-noise and prediction," NASA Reference Publication 1218, July 1989.

[11] A. Tadamasa and M. Zangeneh, "Numerical prediction of wind turbine noise," Renewable Energy, vol. 36, pp. 1902-1912, 2011.

[12] M. J. Lighthill, "On Sound generated aerodynamically I. General theory," Roy Soc London Ser A, vol. 211, pp. 564-87, 1952.

[13] J. E. F. Williams and D. L. Hawkings, "Sound generation by turbulence and surfaces in arbitrary motion," Philos Transact A Math Phys Eng Sci, vol. 264, pp. 321-42, 1969.

[14] K. S. Brentner and F. Farassat, "An analytical comparison of the acoustic analogy and Kirchhoff formulations for moving surfaces," AIAA Journal, vol. 36, no. 8, pp. 1379-1386, 1998.

[15] K. S. Brentner and F. Farassat, "Modeling aerodynamically generated sound of helicopter rotors," Progress in Aerospace Sciences, vol. 39, pp. 83-120, 2003.

[16] STAR-CCM+ Version 8.02 User guide, 2013 CD-adapco.

[17] E. P. N. Duque, M. D. Burklund, and W. Johnson, "Navier-Stokes and comprehensive analysis performance predictions of the NREL phase VI experiment," Journal of Solar Energy Engineering, vol. 125, pp. 457-467, 2003.

[18] C. Tongchitpakdee, S. Benjanirat, and L. N. Sankar, "Numerical simulation of the aerodynamics of horizontal axis wind turbines under yawed flow conditions," in Proc. 43th AIAA Aerospace Sciences Meeting and Exhibit, Reno, NV, AIAA, 2005, pp. 281-304.

[19] N. Sezer-Uzol and N. L. Long, "3-D time-accurate CFD simulations of wind turbine rotor flow fields," in Proc. 44th AIAA Aerospace Sciences Meeting and Exhibit, Reno, NV, AIAA, 2006, p. 394.

[20] J. O. Mo and Y. H. Lee, "CFD investigation on the aerodynamic characteristics of a small-sized wind turbine of NREL PHASE VI operating with a stall-regulated method," Journal of Mechanical Science and Technology, vol. 26, no. 1, pp. 81-92, 2012.

[21] Y. Li, K. J. Paik, T. Xing, and P. M. Carrica, "Dynamic overset CFD simulations of wind turbine aerodynamics," Renewable Energy, vol. 37, no. 1, pp. 285-298, 2012

[22] M. M. Yelmule and E. Anjuri, "CFD predictions of NREL phase VI rotor experiments in NASA/AMES wind tunnel," International Journal of Renewable Energy Research, vol. 3, pp. 250-260, 2013.

[23] R. Lanzafame, S. Mauro, and M. Messina, "Wind turbine CFD modeling using a correlation-based transitional model," Renewable Energy, vol. 52, pp. 31-39, 2013.

[24] R. Lanzafame and M. Messina, "Bem theory: How to take into account the radial flow inside of a 1-D numerical code," Renewable Energy, vol. 39, pp. 440-446, 2012. 


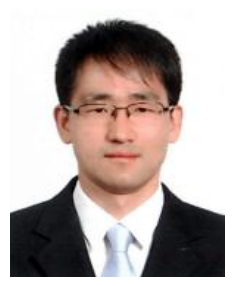

Kyoungsoo Lee received his BS, MS, PhD degrees in the Department of Architectural Engineering of Inha University, Incheon, South Korea. He is working for the CEES, Prairie View A\&M University, Prairie View, Texas, USA as a post doc. researcher. He was a research professor in the Department of Civil \& Environmental Engineering, KAIST in South Korea. His professional areas are the structural engineering and design, CFD, FSI and impact \& blast simulation. Currently, he is focusing on the developing the sound noise simulation for the wind blade. Dr. Lee is a member of AIK, KSSC in South Korea.

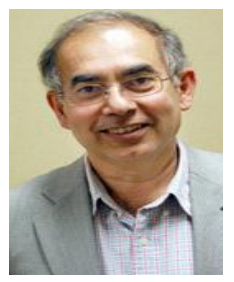

Ziaul Huque received his BS degree in mechanical engineering from Bangladesh University of Engineering and Technology, Bangladesh, the MS degree in mechanical engineering from Clemson University, USA and the Ph.D. degree in mechanical engineering from Oregon State University, USA. He is currently a professor in the Department of Mechanical Engineering and the director of Computational Fluid Dynamics Institute at Prairie View A\&M University. His current research interests are wind turbine noise reduction, fluid-structure interaction, propulsion, inlet-ejector system of rocket based combined cycle engines, clean coal technology, self-propagating high-temperature synthesis. $\mathrm{He}$ received several excellences in teaching and service awards from Roy G. Perry College of Engineering, Lockheed-Martin Tactical Aircraft Systems Teaching Excellence Award, Welliver Summer Faculty Fellowship from Boeing in 2002 and NASA Summer Faculty Fellowship in 2003.

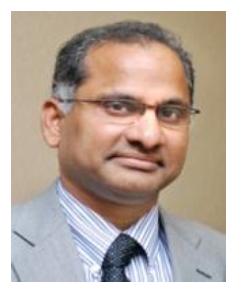

Raghava Kommalapati received his B.Tech degree in civil engineering and M.Tech degree in engineering structures from India. He received the MS and $\mathrm{PhD}$ degrees in civil engineering (environmental engineering) form Luisiana State University, Baton rouge, LA, USA in 1994 and 1995 respectively. Dr. Kommalapati is the principal investigator and director of the Center for Energy and Environmental Sustainability, a NSF funded center. He is also a professor in the Civil \& Environmental Engineering Department. He served as the interim department head of Civil \& Environmental Engineering Department for 3.5 years from January 2010 to August 2013. He is a registered professional engineering (PE) in the State of Texas and a board certified environmental engineer (BCEE). His major field of study is environmental engineering with particular focus on energy and environmental sustainability and air quality. He is an author or editor of books, and has published more than 35 peer-reviewed journal articles and more than 90 proceedings and presentations at regional, national and international conferences. $\mathrm{He}$ is a member of several professional organizations including, AEESP, AAEES, ACS, ASCE, ASEE and honor societies of Tau Beta Phi, Phi Kappa Phi and Sigma Xi.

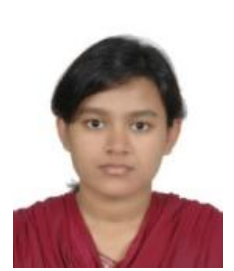

Shrabanti Roy received her BS degree in mechanical engineering from Military Institute of Science \& Technology, Bangladesh, 2011. She is currently a master student at Prairie View A \& M University, Texas and also works under the Center for Energy \& Environmental Sustainability (CEES). Her major concentration is on mechanical engineering and interest area is CFD, thermo fluid science and wind energy.

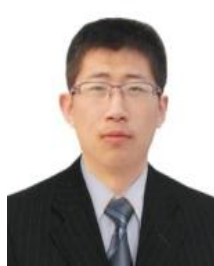

Chao Sui received his BS degree in mechanical engineering from Shan Dong University of Technology, China, 2013. He is currently chasing the master degree of mechanical engineering at Prairie View A \& M University, Texas, USA. He also works under the Center for Energy and Environmental Sustainability (CEES). Chao Sui's research interests are optimization of wind turbine blade, CFD and thermodynamics.

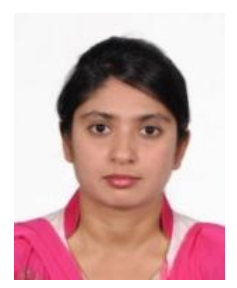

Nazia Binte Munir received her BS degree in mechanical engineering from Military Institute of Science and Technology (MIST), Bangladesh, 2011. She is currently a master student at Prairie View A\&M University, Texas, USA and working under the Cente for Energy and Environmental Sustainability (CEES). Her major concentration is on mechanical engineering and her research interests are CFD, wind energy, thermal engineering. 